\title{
Nutrição e COVID-19: uma investigação de aspectos nutricionais e sua importância
}

\section{diante da pandemia}

\author{
Nutrition and COVID-19: an investigation of nutritional aspects and their importance in health in
}

the view of the pandemic

Nutrición y COVID-19: una investigación de aspectos nutricionales y su importancia en salud ante

la pandemia

Recebido: 27/11/2021 | Revisado: 04/12/2021 | Aceito: 10/12/2021 | Publicado: 18/12/2021

Julia Rocha Rocca

ORCID: https://orcid.org/0000-0001-6791-8818 Centro Universitário de Brasília, Brasil E-mail: julia.rocca@sempreceub.com Emilly Menezes de Carvalho ORCID: https://orcid.org/0000-0001-7973-6595 Centro Universitário de Brasília, Brasil

E-mail: emilly.menezes@ sempreceub.com Dayanne da Costa Maynard ORCID: https://orcid.org/0000-0002-9295-3006 Centro Universitário de Brasília, Brasil E-mail: day_nut@yahoo.com.br

Michele Ferro de Amorim Cruz ORCID: https://orcid.org/0000-0003-0387-1509 Centro Universitário de Brasília, Brasil E-mail: micheleferro.ac@gmail.com

\begin{abstract}
Resumo
Diante da pandemia causada pelo COVID-19, a população de todo o mundo se viu frente a uma nova realidade com incertezas, medo e dificuldades. Este novo estilo de vida perante o isolamento social e quarentena levou a mudanças comportamentais com prejuízos para a saúde física e mental. É sabido da importância de um sistema imunológico regulado como forma de barreira contra infecções que podem acometer o nosso corpo, e que, a alimentação é uma das principais formas de adquirir nutrientes necessários para essa regulação da imunidade. Portanto, este trabalho buscou investigar a influência de aspectos nutricionais e sua importância para saúde no enfrentamento da pandemia do COVID-19. Foram selecionados artigos científicos publicados entre os anos de 2019 a 2021. Nestes estudos foi possível analisar e ressaltar a importância dos micronutrientes que, em adequação no organismo ou em terapia com dosagens elevadas melhoram a inflamação da doença e consequentemente os prognósticos dos pacientes. Mostra-se a importância de mais estudos que avaliem terapias utilizando micronutrientes no tratamento COVID 19.
\end{abstract}

Palavras-chave: COVID-19; Alimentação; Imunidade; Nutrientes.

\begin{abstract}
In front of the pandemic caused by COVID-19, the whole world was seen with a new reality of uncertainty, fear, and difficulties. This new lifestyle in front of social isolation and quarantine led to behavioral changes with damage to physical and mental health. It is known of the importance of a regulated immune system as a barrier against infections that can attack our body, and that food is one of the main ways to acquire nutrients necessary for this regulation of immunity. Therefore, this work sought to investigate the influence of nutritional aspects and your importance for health in confronting with COVID-19 pandemic. Scientific articles published between the years 2019 to 2021 were selected. In these studies, it was possible to analyze and emphasize the importance of micronutrients that, inadequacy in the body or therapy with high dosages, improve the inflammation of the disease and, consequently, the prognosis of patients. Turn up the importance of further studies evaluating therapies used micronutrients in COVID-19 treatment.
\end{abstract}

Keywords: COVID-19; Food; Immunity; Nutrients.

\section{Resumen}

Delante de la pandemia causada por COVID-19, la población de mundo el todo se enfrente de a una nueva realidad 
con incertidumbre, miedo y dificultades. Este nuevo estilo de vida frente al aislamiento social y la cuarentena causó cambios de comportamiento con daños a la salud física y mental. Es sabido la importancia de un sistema inmunológico regulado como obstáculo frente a las infecciones que pueden afectar a nuestro organismo, y que la alimentación es una de las principales maneras de adquirir los nutrientes necesarios para esta regulación de la inmunidad. Por tanto, este trabajo busca investigar la influencia de los aspectos nutricionales y su importancia para la salud en el afrontamiento de la pandemia de COVID-19. Se seleccionaron artículos científicos publicados entre los años 2019 a 2021. En estos estudios es posible analizar y enfatizar la importancia de los micronutrientes que, en adecuación en el organismo o en terapia con altas dosis, mejorar la inflamación de la enfermedad y, en consecuentemente, el pronóstico de los pacientes. Se muestra la importancia de nuevos estudios que evalúen las terapias que utilizan micronutrientes en el tratamiento con COVID-19.

Palabras clave: COVID-19; Alimentación; Inmunidad; Nutrientes.

\section{Introdução}

No final do ano de 2019 surgiram os primeiros relatos de casos de uma nova pneumonia, até então desconhecida, na cidade de Wuhan, província de Hubei, na China (Zhou et al., 2020). Essa doença, denominada COVID-19 pela Organização Mundial da Saúde (OMS) (Pascarella et al., 2020), se espalhou rapidamente pelo mundo e, alguns meses depois, em 30 de Janeiro de 2020 a OMS declarou este surto como uma Emergência de Saúde Pública de Importância Mundial (Albuquerque et al., 2020).

Diante da nova realidade vivida frente à pandemia do Coronavírus, pessoas do mundo todo passaram a viver em isolamento social, o que trouxe novos hábitos de vida e consequentemente, alimentares. Estudos demonstram maiores números de preparação de refeições no ambiente familiar e em contraposição, aumento também no consumo de comidas ultra processadas, fast foods e bebidas alcoólicas (Aro et al., 2021; Maynard et al., 2020). Além disso, o novo estilo de vida adaptado ao isolamento social acarretou em alterações psicológicas relacionadas a estresse e ansiedade, com influência direta nas escolhas alimentares e consequentemente, na ingestão de nutrientes (Aro et al., 2021).

A doença causada pela COVID-19 é considerada crítica e associa-se a um grande estresse catabólico no organismo, gerando complicações que podem levar à morte. As manifestações clínicas podem ser nulas em $80 \%$ dos casos, segundo a OMS, e quando aparecem, são sintomas gripais já conhecidos, dentre eles febre $(88,7 \%)$, tosse $(67,8 \%)$, fadiga $(38,1 \%)$, produção de escarro $(33,4 \%)$, falta de ar $(18,6 \%)$, dor na garganta $(13,9 \%)$ e dor de cabeça $(13,6 \%)$ (Albuquerque et al., 2020$)$. Alguns estudos demonstram que, além destes sintomas mais comuns, ainda existem comprometimentos neurológicos que podem ser observados como a perda de olfato (anosmia), perda de paladar (ageusia), tontura e fraqueza muscular em $34,6 \%$ dos casos (Gama \& Cavalcante, 2020), que podem influenciar diretamente no estado nutricional do indivíduo.

Uma série de práticas são recomendadas para auxiliar no combate contra infecções virais respiratórias, como a lavagem de mãos e o distanciamento de pessoas com sintomas gripais, entretanto há a necessidade de abordar a importância da alimentação saudável e equilibrada a fim de melhorar o estado nutricional, fortalecer a imunidade e diminuir o acometimento de contágios virais. É evidente que a presença de alguns nutrientes em estado de deficiência no organismo pode trazer prejuízos à saúde e piora na defesa contra estas infecções (Calder et al., 2020). Estudos demonstram que o estado nutricional irá influenciar na condição clínica e sabe-se que os nutrientes contribuem no papel dos efeitos imunomoduladores do organismo. Sendo assim, é visto a importância de uma alimentação equilibrada que atenda às necessidades nutricionais, influenciando na imunidade e até mesmo diminuindo a ocorrência de infecções e reinfecções (Richardson \& Lovegrove, 2020; Massarollo et al., 2021).

Com base na realidade hoje observada em todo o mundo diante da pandemia causada pelo novo coronavírus e, sabendo da relação que a nutrição tem diretamente com promoção, manutenção e recuperação da saúde, é de extrema relevância investigar a influência do comportamento alimentar e do consumo de nutrientes na melhora do estado nutricional e 
sua importância diante da pandemia do COVID-19, para que, além de enriquecer as bases científicas que ainda são limitadas acerca deste novo vírus, seja capaz de contribuir e agregar, tanto no benefício da saúde da população como um todo e na melhora do prognóstico de indivíduos acometidos por essa doença, quanto para orientação e auxílio de profissionais de saúde em relação às condutas referentes à alimentação. Fundamentado no que foi exposto, este trabalho buscou investigar a influência de aspectos nutricionais e sua importância para saúde no enfrentamento da pandemia do COVID-19.

\section{Metodologia}

O estudo foi feito por meio de uma revisão integrativa da literatura sobre aspectos nutricionais e sua importância para a saúde diante da pandemia do COVID-19, abordando os nutrientes específicos: vitamina A, C, D, E e selênio. Foi realizado com base em artigos científicos e periódicos da área da saúde, disponíveis nos idiomas inglês e português, entre os anos de 2019 e 2021.

Foram utilizados como descritores de saúde: COVID-19, Sistema Imune/Immune System, Doença/Disease, Alimentação/Food, Ingestão de alimentos/Eating, Nutrientes/Nutrients, Pandemia/Pandemics, Comportamento alimentar/Feeding Behavior, todos os descritores cadastrados nos descritores de ciências da saúde (DECS). Os dados coletados foram encontrados por meio das bases de dados eletrônicos Scielo, Pubmed e, utilizou a literatura cinzenta Google Acadêmico.

\section{Análise de dados}

Este estudo de revisão abordou artigos previamente selecionados pelo título, após a seleção do mesmo foi lido o resumo e em seguida, o artigo na íntegra. A pesquisa se iniciou buscando estudos que realizaram intervenção nutricional em pacientes com COVID-19, obtendo sucesso com os nutrientes vitamina D e C. Em seguida, buscou-se estudos que avaliassem os níveis plasmáticos das vitaminas e os correlacionassem com os sintomas e possíveis melhorias no tratamento da doença, obtendo êxito com os nutrientes vitamina A e Selênio. Foram incluídos manuscritos que tratavam sobre os aspectos nutricionais no período da pandemia do COVID-19, dentre eles artigos de revisão que abordavam sobre o tema da presente pesquisa e estudos de campo que testavam intervenções nutricionais em pacientes com COVID-19, com maior número de publicações entre os anos de 2019 a 2021. Foram excluídos os artigos que não abordaram sobre nutrição, consumo de nutrientes, comportamento alimentar diante da pandemia do COVID-19 e imunidade.

Após prévia escolha e leitura crítica dos estudos, foram identificados os fundamentos de relevância em cada texto e posterior agrupamento de subtemas que sintetizam as produções.

\section{Resultados e Discussão}

A busca dos artigos nas bases de dados ocorreu entre os meses de agosto e setembro de 2021. Mediante os critérios de inclusão e exclusão de artigos, foram selecionados 5 artigos científicos para tratar de forma específica o assunto deste estudo (Figura 1). Pesquisas recentes têm demonstrado a importância da nutrição na recuperação da saúde e redução de sintomas da patologia de COVID-19, dados apresentados no Quadro 1. 
Figura 1. Fluxograma do levantamento de dados realizados para a presente revisão.

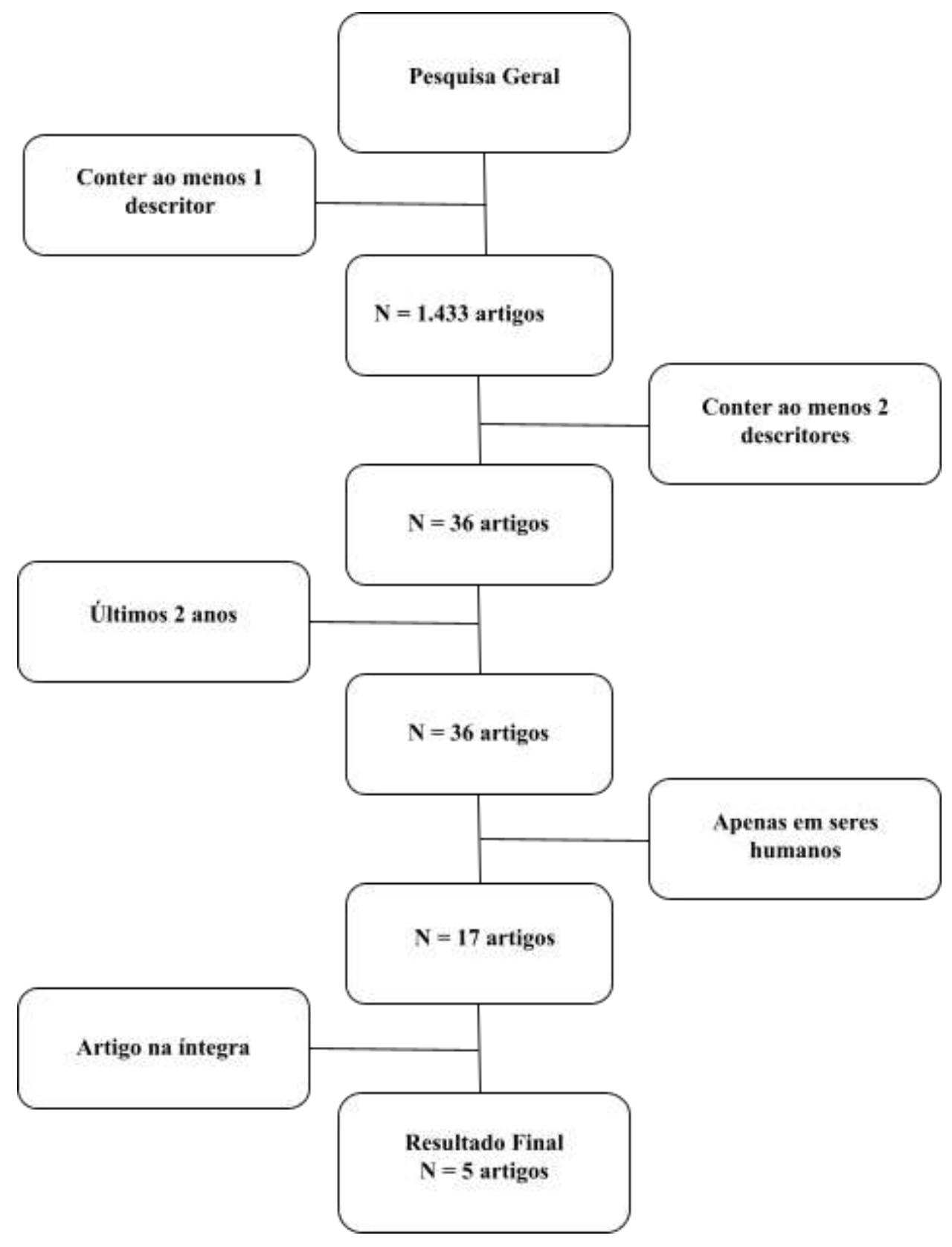

Fonte: Autores.

\subsection{Aspectos nutricionais e a relação com o COVID-19}

Os nutrientes exercem um papel importante na imunomodulação, sendo assim dependem de adequações para desempenharem seus papéis na defesa do organismo. As depleções desenvolvem-se progressivamente ao longo do tempo, antes de manifestar sintomas o corpo se adapta por meio da redução de modulações bioquímicas, comprometimento das vias e funções metabólicas e a diminuição dos estoques de nutrientes. Essas deficiências nutricionais são capazes de reduzir a resistência contra as infecções, e até mesmo reinfecções, portanto a defesa imunológica depende do aporte adequado de nutrientes para a prevenção de infecções e um melhor prognóstico da COVID-19 (Richardson \& Lovegrove, 2020).

\section{Vitamina A}

A Vitamina A é uma vitamina lipossolúvel formada a partir da ingestão de alimentos que contenham seus precursores 
de formação. As principais fontes alimentícias de origem animal incluem leites e derivados, ovos e fígado. De origem vegetal são fontes os alimentos ricos em carotenoides, principalmente betacarotenos, podem ser identificados como os alimentos que possuem coloração amarela e alaranjadas e vegetais verde escuros, incluem por exemplo cenoura, espinafre, manga, couve, beterraba, entre outros. Para que seja adequadamente absorvida, essa vitamina depende da ingestão conjunta de lipídeos na dieta. Este micronutriente possui três funções principais realizadas no organismo sendo elas, a composição do grupo prostéticos (elementos não proteicos ligados a uma proteína) de pigmentos da visão, a síntese de glicoproteínas hidrofóbicas e hormônio com ação no controle de proliferação e diferenciação celular. Além disso, a vitamina A também age na produção de muco e resistência às infecções, mediada pela ação moduladora da resposta imune, importante papel na imunidade (Cozzolino, 2020).

A COVID-19 é uma doença motivada pela nova Síndrome Respiratória Aguda Grave do Coronavírus 2, denominada SARS-CoV-2, que possui alto grau de morbidade e mortalidade em todo o mundo (Li et al., 2020). Essa doença causa danos pulmonares e hiper inflamação no organismo, e dentre as principais manifestações clínicas pode-se citar a pneumonia grave, a insuficiência respiratória e a morte. Os piores resultados clínicos são relacionados a desregulação nas respostas imunes que leva ao descontrole de liberação de citocinas pró inflamatórias no organismo (Tepasse et al., 2021).

A vitamina A possui importante papel no desenvolvimento de tecido pulmonar e recuperação de tecido após lesões por infecções, podendo assim ser associada a melhora na recuperação de indivíduos acometidos com pneumonia pós COVID19 grave e ainda afeta de forma positiva a resposta imune inata e adaptativa. Foi observado que indivíduos acometidoscom a doença em fase aguda possuem níveis plasmáticos de vitamina A consideravelmente mais baixos em comparação com indivíduos em fase de recuperação da mesma. Além disso, baixos níveis de vitamina A plasmáticos foram associados com parâmetros inflamatórios elevados como proteína $\mathrm{C}$ reativa, albumina e ferritina. A diminuição dos níveis plasmáticos de vitamina A $<2$ mg mostrou aumento na taxa de desenvolvimento de Síndrome do Desconforto Respiratório Agudo (SDRA) e mortalidade (Tepasse et al., 2021).

\section{Vitamina C}

A vitamina C, também chamada de ácido ascórbico e ascorbato, é encontrada predominantemente em alimentos de origem vegetal como por exemplo na laranja, no kiwi, no mamão, no morango e na goiaba, embora suas concentrações possam variar de acordo com estação do ano, cocção e tempo de armazenamento destes alimentos. Dentre as funções destes nutrientes, podemos citar a relevância de sua capacidade em agir na imunidade. Por ser uma vitamina hidrossolúvel e devido sua facilidade em doar elétrons, ela age na linha de frente de defesa do organismo com importante papel antioxidante. Além disso, possui relevância também no auxílio da absorção de ferro no organismo (Cozzolino, 2020).

A piora da sintomatologia do COVID-19 após duas semanas está relacionada ao dano causado no sistema imunológico e não à replicação do vírus instaurado. Isso demonstra que a terapia antiviral, de forma isolada, é insuficiente parao tratamento desses pacientes (Zhang et al., 2020). Sabe-se que a vitamina C possui um forte poder antioxidante e atua diretamente contra os radicais livres, desempenhando assim um papel relevante na melhora dos efeitos da inflamação, reduzindo a produção de citocinas pró inflamatórias e agindo na imunorregulação (Zhang et al., 2020).

Em um estudo randomizado, foi efetuada a terapia de infusão intravenosa de altas doses de vitamina $\mathrm{C}$ em pacientes críticos com COVID-19, como resultado concluiu-se que essa vitamina obteve um potencial de benefício na oxigenação destes pacientes, porém sem melhora na ventilação mecânica (Zhang et al., 2020). 


\section{Vitamina D}

A vitamina D, também chamada de calciferol, é um pré-hormônio lipossolúvel que em conjunto com o paratormônio (PTH) exerce suas principais funções, regulando a homeostase do cálcio e do metabolismo ósseo. É encontrada na forma de vitamina D2, ergocalciferol e vitamina D3, colecalciferol. Sua principal fonte é obtida através dos raios UVB da luz solar. Estima-se que a exposição solar sintetiza cerca de 80 a $100 \%$ das necessidades dos indivíduos. No entanto, também está presente em alimentos como óleo de fígado de bacalhau e peixes gordurosos (salmão, atum e cavala), ovos, leite e cereais (Maeda et al., 2014). A ausência da exposição solar e a baixa ingestão de alimentos ricos, leva a uma necessidade de suplementação, o que é frequentemente necessário, pois a exposição e a ingesta normalmente são insuficientes. Vale ressaltar a influência de alguns fatores que interferem na absorção dessa vitamina como pigmentação da pele, idade, estação do ano, condições climáticas e local de trabalho (Richardson \& Lovegrove, 2020).

Atualmente, esse nutriente vem sendo estudado e relacionado a várias patologias como doenças vasculares, inflamatórias, em neoplasias e doenças neurodegenerativas (Herrmann et al., 2016). Indícios mostram também sua relação com a saúde respiratória, abrangendo as respostas inflamatórias pulmonares e no aumento dos mecanismos da defesa inata contra os patógenos inflamatórios. É apontada sua atuação nos processos imuno modulantes, anti-inflamatórios e antiinfecções (Richardson \& Lovegrove, 2020).

Um ensaio clínico realizado com pacientes com diagnóstico positivo para COVID-19 demonstrou a presença de melhores resultados em relação aos sintomas de tosse e perda sensorial gustativa em indivíduos submetidos a terapia de vitamina D contendo 5000 UI de vitamina D3, composta de 125 ug de colecalciferol durante 14 dias, em comparação a um grupo controle que foi submetido com menores doses de vitamina D (Sabico et al., 2021).

Outro estudo realizado com pacientes acima de 18 anos, portadores de COVID-19, com hipovitaminose D e doença de grau leve a moderado, foi analisado os níveis séricos de vitamina D e os marcadores inflamatórios em dois grupos randomizados. Foi observado que a terapia adjuvante de vitamina D associada ao tratamento padrão para COVID-19 melhorou os níveis séricos do marcador desta vitamina e diminuiu de forma significativa os marcadores inflamatórios (Lakkireddy et al., 2021).

Quando relacionada à imunidade, a vitamina $\mathrm{D}$ realiza um papel fundamental na modulação do sistema imunológico.Seu receptor está presente nas células imunológicas, como células dendríticas, linfócitos B e T, monócitos e macrófagos, atuando também na manutenção da integridade da barreira imune e as respostas subsequentes das células imunes adaptativas. Quando em deficiência, está também relacionada a desregulação do estado inflamatório e ao mau funcionamento do sistema imune (Galmés et al., 2020).

\section{Vitamina $E$}

A vitamina E é representada por oito tipos de isoformas, sendo quatro tocoferóis e quatro tocotrienóis, além de ser lipossolúvel, ou seja, dependente de uma lipoproteína para ser transportada e metabolizada, regulada e excretada no fígado (Jovic et al., 2020). A principal fonte desse nutriente é de forma exógena, através de alimentos ricos como os óleos vegetais (soja, girassol, milho, noz, semente de algodão, palma e gérmen de trigo). A vitamina E possui duas principais funções, a primeira é agindo como antioxidante, eliminando radicais livres e detendo a oxidação de ácidos graxos poli-insaturados e a segunda é agindo na regulação de enzimas que atuam na transdução de sinal, associada também na inibição da atividade da proteína quinase (PKC) (Lee \& Han, 2018).

Quando relacionada ao seu papel antioxidante, ela protege a membrana celular contra prejuízos oxidativos, apoia a integridade das barreiras epiteliais respiratórias, aumenta a atividade das células Natural Killer (NK) e diminui a produção de prostaglandina E2, modulando a produção de IFN-y e interleucina 2 entre outras funções mediadoras do sistema imunológico. 
Quando em deficiência, prejudica as funções da imunidade, favorecendo o acometimento de infecções. Portanto, a suplementação desta vitamina pode melhorar o sistema imunológico e diminuir as infecções no trato respiratório (Gasmi et al., 2020).

Em um experimento feito em ratos com SDRA, foi realizado introdução com ácido oleico, em 42 ratos machos, divididos em sete grupos. Um grupo controle (saudável), um grupo de AO (ácido oleico) e os demais em administração de antioxidantes, como ácido lipóico, vitamina $\mathrm{C}$, vitamina $\mathrm{E}$, metilprednisolona em baixa dose e o outro em alta dose, na forma cristalina e dissolvida em água destilada e a metilprednisolona em pó e dissolvida em solução salina. Neste estudo concluiu-se que as vitaminas antioxidantes podem ser úteis para a pré-medicação de SDRA (Erol et al., 2019).

\section{Selênio}

O selênio é um oligoelemento, sendo também um metaloide, da mesma família do oxigênio e do enxofre. É encontrado na forma orgânica, sendo as principais formas a selenometionina, selenocisteína e na forma inorgânica como selenito, seleneto, selenato e o selênio. As principais fontes dietéticas são grãos, vegetais, frutos do mar, carnes, laticínios e nozes. É também encontrado no solo e são os principais componentes dos suplementos alimentares (Santos et al., 2018). A dosagem pode variar de acordo com o solo em que o alimento foi cultivado, em solos seminíferos possuem dosagens mais altas (Mehdi et al., 2013).

Os níveis adequados desse oligoelemento são importantes, pois realizam diversas funções no organismo, o que inclui sistema nervoso central, reprodução, sistema endócrino, muscular, sistema cardiovascular e na imunidade (Avery \& Hoffmann, 2018). A sua deficiência aumenta a virulência e a progressão de infecções virais. No sistema imunológico realiza diversas funções, como regulação da resposta das células T CD4 +, atua também nas células efetoras citotóxicas (T CD8 +), nas células NK e na produção de anticorpos, entre outras funções (BAE; KIM, 2020).

Em um estudo transversal realizado em pacientes com COVID-19, foi feito retirada de sangue e armazenadas a $-80^{\circ}$, para avaliação em laboratório. Foi observado que pacientes com déficit de selênio acometidos com COVID-19 possuem maiores riscos de mortalidade (Moghaddam et al., 2020). 
Quadro 1. Principais resultados sobre os aspectos nutricionais e a relação com a COVID-19.

\begin{tabular}{|c|c|c|c|c|}
\hline Nutriente & Estudo & Tipo de estudo & Principais resultados & Autores \\
\hline Vitamina A & $\begin{array}{l}\text { Vitamin A plasma levels in } \\
\text { COVID-19 patients: a } \\
\text { prospective multicenter study } \\
\text { and hypothesis. }\end{array}$ & $\begin{array}{l}\text { Transversal } \\
\text { observacional, } \\
\text { multicêntricoe } \\
\text { prospectivo. }\end{array}$ & $\begin{array}{l}\text { Indivíduos em fase aguda da COVID } 19 \\
\text { apresentam níveis mais baixos de vitamina A } \\
\text { em comparação a indivíduos em recuperação } \\
\text { da doença. } \\
\text { Baixos níveis de vitamina A foram associados } \\
\text { aos parâmetros inflamatórios mais elevados e } \\
\text { aumento na taxa de desenvolvimento de SDRA } \\
\text { e mortalidade. }\end{array}$ & $\begin{array}{c}\text { Tepasse etal., } \\
2021 .\end{array}$ \\
\hline Vitamina C & $\begin{array}{l}\text { Pilot trial of high-dose vitamin } \\
C \text { in critically ill COVID-19 } \\
\text { patients. }\end{array}$ & $\begin{array}{l}\text { Ensaio clínico } \\
\text { randomizado. }\end{array}$ & $\begin{array}{l}\text { Terapia antiviral de forma isolada não é } \\
\text { suficiente para tratar pacientes com COVID } \\
\text { 19. A terapia de infusão intravenosa de altas } \\
\text { doses de vitamina C melhoram a oxigenação } \\
\text { destes pacientes. }\end{array}$ & $\begin{array}{l}\text { Zhang et al., } \\
2020 .\end{array}$ \\
\hline \multirow[t]{2}{*}{ Vitamina D } & $\begin{array}{l}\text { Effects of a 2-Week } 5000 \mathrm{IU} \\
\text { versus } 1000 \text { IU Vitamin D3 } \\
\text { supplementation onrecovery of } \\
\text { symptoms in patients with mild } \\
\text { to moderate Covid-19: A } \\
\text { randomized clinical trial } \\
\end{array}$ & $\begin{array}{l}\text { Ensaioclínico } \\
\text { randomizado. }\end{array}$ & $\begin{array}{l}\text { Melhora nos sintomas de tosse e perda } \\
\text { sensorial gustativa em indivíduos submetidos } \\
\text { a maiores doses de terapia de vitamina } D \text {. }\end{array}$ & $\begin{array}{c}\text { Sabico et al., } \\
2021 .\end{array}$ \\
\hline & $\begin{array}{l}\text { Impact of daily high dose oral } \\
\text { vitamin D therapy on the } \\
\text { inflammatory markers in } \\
\text { patients with COVID } 19 \\
\text { disease. }\end{array}$ & $\begin{array}{l}\text { Ensaio clínico } \\
\text { intervencional, } \\
\text { prospectivo, } \\
\text { randomizado, aberto. }\end{array}$ & $\begin{array}{l}\text { A terapia adjuvante de vitamina D associada } \\
\text { ao tratamento padrão para COVID-19 } \\
\text { melhorou os níveis séricos do marcador desta } \\
\text { vitamina e diminui de forma significativa os } \\
\text { marcadores inflamatórios destes indivíduos, } \\
\text { em comparação ao grupo que recebeu apenas } \\
\text { o tratamento padrão da doença. }\end{array}$ & $\begin{array}{l}\text { Lakkireddy, } \\
\text { et al., } 2021 .\end{array}$ \\
\hline Selênio & $\begin{array}{l}\text { Selenium deficiency is } \\
\text { associated with mortality risk } \\
\text { from COVID-19. }\end{array}$ & Estudo transversal. & $\begin{array}{l}\text { Pacientes acometidos com COVID } 19 \text { que } \\
\text { apresentam déficit de Selênio, possuem } \\
\text { maiores riscos de mortalidade. }\end{array}$ & $\begin{array}{l}\text { Moghaddam } \\
\text { et al., } 2020 .\end{array}$ \\
\hline
\end{tabular}

Fonte: Autores.

Em consequência à gravidade da doença causada pelo novo coronavírus, $30 \%$ dos pacientes acabam necessitando do suporte respiratório e hemodinâmico urgente em unidades de terapia intensiva (UTI), devido ao quadro de SRDA permanecendo assim um período longo de internação. Esse período de internação prolongado apresenta um risco para o surgimento de desnutrição e de perda de massa muscular desses pacientes, devido a alguns fatores afetados por consequência da doença que interferem na ingestão alimentar do paciente, como a falta de ar, a perda de olfato e paladar e boca seca. Portanto, é de extrema importância a avaliação nutricional e o manejo precoce dos cuidados nutricionais como suporte a evitar o mau prognóstico desses indivíduos (Holdoway, 2020).

Devido a vulnerabilidade do público composto de pacientes da UTI com COVID-19 (pacientes idosos, portadores de DCNT) e com ingestão alimentar reduzida, e ao tempo prolongado de internação, há a indicação de suporte nutricional de nutrição enteral para o alcance das necessidades nutricionais desses pacientes. Porém, evidências demonstram que indivíduos com sintomas gastrointestinais não devem ser abordados para indicação deste tipo de terapia, e nesses casos, a nutrição parenteral deve ser aconselhada para suporte suplementar ou total destes pacientes (Thibault et al., 2020).

Em um estudo feito em pacientes com COVID-19 em uso de terapia enteral, geralmente com uma fórmula com $75 \mathrm{~g}$ de proteína/L e 1,5 kcal/ml, foi avaliado o suporte nutricional de acordo com o total de energia e proteína fornecido e dividido pelo IBW (idade, sexo, altura e peso corporal ideal). Como resultado foi sugerido que o suprimento de meta a ser utilizado de proteína >0,8g/IBW/dia pode estar associado à redução da mortalidade de pacientes em UTI (Silvah et al., 2021).

Diante do exposto, pode-se observar a importância de uma triagem nutricional de qualidade em âmbito hospitalar a fim de diagnosticar desnutrição nos pacientes. Nos enfermos acometidos com COVID-19, esse diagnóstico passa a ser ainda mais importante devido a gravidade da doença e para que seja realizada terapia nutricional alternativa por via enteral ou 
parenteral, quando necessárias, em metas energéticas e proteicas adequadas que se mostram relevantes na melhora destes indivíduos.

\section{Conclusão}

A pandemia e as alterações no comportamento social da população neste período trouxeram mudanças no padrão alimentar da sociedade com potencial de danos na saúde como um todo. Visto que o consumo adequado de nutrientes é primordial para a regulação da imunidade, foi observado que o consumo adequado de vitamina A e selênio são capazes de melhorar o estado nutricional de pacientes acometidos com COVID-19, bem como gerar um prognóstico positivo, reduzir a mortalidade e diminuir a taxa de desenvolvimento de SDRA, porém sem resultados que avaliassem o uso destes nutrientes no tratamento da doença. Observou-se também que terapias utilizando altas doses de vitamina D e C são parâmetros de grande influência na melhora da sintomatologia de pacientes acometidos com o vírus e também capazes de melhorar a oxigenação dos mesmos.

Entretanto, mostra-se necessário um maior número de estudos que utilizem micronutrientes em terapias para tratamento da COVID-19. É visto na literatura uma escassez de ensaios clínicos que abordam vitamina A, E e selênio na terapia dos pacientes acometidos com essa doença, considerando o impacto positivo destes nutrientes na imunidade e na saúde já comprovados. Sugerimos novos estudos utilizando estes nutrientes no tratamento em seres humanos acometidos com COVID-19 para enriquecimento da literatura.

\section{Referências}

Albuquerque, L. P., da Silva, R. B., \& de Araújo, R. M. S. (2020). COVID-19: origin, pathogenesis, transmission, clinical aspects and current therapeutic strategies. Revista Prevenção de Infecção e Saúde, 6. https://doi.org/10.26694/repis.v6i0.10432

Ammar, A., Brach, M., Trabelsi, K., Chtourou, H., Boukhris, O., Masmoudi, L., \& ECLB-COVID19 Consortium. (2020). Effects of COVID-19 home confinement on eating behaviour and physical activity: results of the ECLB-COVID19 international online survey. Nutrients, $12(6)$, 1583. https://doi.org/10.3390/nu12061583

Anjum, S., Ullah, R., Rana, M. S., Ali Khan, H., Memon, F. S., Ahmed, Y., \& Faryal, R. (2020). COVID-19 pandemic: A serious threat for public mental health globally. Psychiatria Danubina, 32(2), 245-250. https://doi.org/10.24869/psyd.2020.245

Aro, F., Pereira, B. V., Navarro, D., \& Bernardo, A. (2021). Comportamento alimentar em tempos de pandemia por Covid-19. Brazilian Journal of Development, 7(6), 59736-59748. https://doi.org/10.34117/bjdv7n6-386

Avery, J. C., \& Hoffmann, P. R. (2018). Selenium, selenoproteins, and immunity. Nutrients, 10(9), 1203. https://doi.org/10.3390/nu10091203

Bae, M., \& Kim, H. (2020). The role of vitamin C, vitamin D, and selenium in immune system against COVID-19. Molecules, 25(22), 5346. https://doi.org/10.3390/molecules25225346

Calder, P. C., Carr, A. C., Gombart, A. F., \& Eggersdorfer, M. (2020). Optimal nutritional status for a well-functioning immune system is an important factor to protect against viral infections. Nutrients, 12(4), 1181. https://doi.org/10.3390/nu12041181

Cozzolino, S. M. F. (2005). Biodisponibilidade de nutrientes. Editora Manole. https://doi.org/10.1590/S1516-93322005000100017

Erol, N., Saglam, L., Saglam, Y. S., Erol, H. S., Altun, S., Aktas, M. S., \& Halici, M. B. (2019). The protection potential of antioxidant vitamins against acute respiratory distress syndrome: a rat trial. Inflammation, 42(5), 1585-1594. https://doi.org/10.1007/s10753-019-01020-2

Fernández-Quintela, A., Milton-Laskibar, I., Trepiana, J., Gómez-Zorita, S., Kajarabille, N., Léniz, A., \& Portillo, M. P. (2020). Key aspects in nutritional management of COVID-19 patients. Journal of clinical medicine, 9(8), 2589. https://doi.org/10.3390/jcm9082589

Gama, B. D. S., \& Cavalcante, K. N. (2020). Pandemia do covid-19: acometimento neurológico e os impactos cerebrais. Brazilian Journal of Health Review, 3(6). https://doi.org/10.34119/bjhrv3n6-285

Galmés, S., Serra, F., \& Palou, A. (2020). Current state of evidence: influence of nutritional and nutrigenetic factors on immunity in the COVID-19 pandemic framework. Nutrients, 12(9), 2738. https://doi.org/10.3390/nu12092738

Gasmi, A., Tippairote, T., Mujawdiya, P. K., Peana, M., Menzel, A., Dadar, M., \& Bjørklund, G. (2020). Micronutrients as immunomodulatory tools for COVID-19 management. Clinical Immunology, 108545. https://doi.org/10.1016/j.clim.2020.108545

Herrmann, M., Farrell, C. J. L., Pusceddu, I., Fabregat-Cabello, N., \& Cavalier, E. (2017). Assessment of vitamin D status-a changing landscape. Clinical Chemistry and Laboratory Medicine (CCLM), 55(1), 3-26. https://doi.org/10.1515/cclm-2016-0264 
Holdoway, A. (2020). Nutritional management of patients during and after COVID-19 illness. British Journal of Community Nursing, 25(Sup8), S6-S10. https://doi.org/10.12968/bjen.2020.25.Sup8.S6

Hossain, M. M., Sultana, A., \& Purohit, N. (2020). Mental health outcomes of quarantine and isolation for infection prevention: a systematic umbrella review of the global evidence. Epidemiology and health, 42. doi: 10.4178 / epih.e2020038

Jovic, T. H., Ali, S. R., Ibrahim, N., Jessop, Z. M., Tarassoli, S. P., Dobbs, T. D., \& Whitaker, I. S. (2020). Could vitamins help in the fight against COVID19?. Nutrients, 12(9), 2550. https://doi.org/10.3390/nu12092550

Lakkireddy, M., Gadiga, S. G., Malathi, R. D., Karra, M. L., Raju, I. P. M., Chinapaka, S., \& Kandakatla, M. (2021). Impact of daily high dose oral vitamin D therapy on the inflammatory markers in patients with COVID 19 disease. Scientific Reports, 11(1), 1-8. https://doi.org/10.1038/s41598-021-97181-y

Lee, G. Y., \& Han, S. N. (2018). The role of vitamin E in immunity. Nutrients, 10(11), 1614. https://doi.org/10.3390/nu10111614

Li, R., Wu, K., Li, Y., Liang, X., Tse, W. K. F., Yang, L., \& Lai, K. P. (2020). Revealing the targets and mechanisms of vitamin A in the treatment of COVID19. Aging (Albany NY), 12(15), 15784. doi: 10.18632/aging.103888

Ma, C., Cong, Y., \& Zhang, H. (2020). COVID-19 and the Digestive System. The American journal of gastroenterology. doi: 10.14309/ajg.0000000000000691

Maeda, S. S., Borba, V. Z., Camargo, M. B. R., Silva, D. M. W., Borges, J. L. C., Bandeira, F., \& Lazaretti-Castro, M. (2014). Recomendações da Sociedade Brasileira de Endocrinologia e Metabologia (SBEM) para o diagnóstico e tratamento da hipovitaminose D. Arquivos Brasileiros de Endocrinologia \& Metabologia, 58, 411-433. https://doi.org/10.1590/0004-2730000003388

Mehdi, Y., Hornick, J. L., Istasse, L., \& Dufrasne, I. (2013). Selenium in the environment, metabolism and involvement in body functions. Molecules, 18(3), 3292-3311. https://doi.org/10.3390/molecules 18033292

Moghaddam, A., Heller, R. A., Sun, Q., Seelig, J., Cherkezov, A., Seibert, L., \& Schomburg, L. (2020). Selenium deficiency is associated with mortality risk from COVID-19. Nutrients, 12(7), 2098. https://doi.org/10.3390/nu12072098

Maynard, D. C., Anjos, H. A., Magalhães, A. C. V., Grimes, L. N., Costa, M. G. O., Santos, R. B. (2020). Consumo alimentar e ansiedade da população adulta durante a pandemia do COVID-19 no Brasil (2020). Research, Society and Development, 9 (11), 1-22, https://doi.org/10.33448/rsd-v9i11.9905.

Pascarella, G., Strumia, A., Piliego, C., Bruno, F., Del Buono, R., Costa, F., \& Agrò, F. E. (2020). COVID-19 diagnosis and management: a comprehensive review. Journal of internal medicine, 288(2), 192-206. https://doi.org/10.1111/joim.13091

Richardson, D. P., \& Lovegrove, J. A. (2021). Nutritional status of micronutrients as a possible and modifiable risk factor for COVID-19: a UK perspective. British journal of nutrition, 125(6), 678-684. https://doi.org/10.1017/S000711452000330X

Sabico, S., Enani, M. A., Sheshah, E., Aljohani, N. J., Aldisi, D. A., Alotaibi, N. H., \& Al-Daghri, N. M. (2021). Effects of a 2-Week 5000 IU versus 1000 IU Vitamin D3 supplementation on recovery of symptoms in patients with mild to moderate Covid-19: A randomized clinical trial. Nutrients, 13(7), 2170. https://doi.org/10.3390/nu13072170

Santos, L. R., Neves, C., Melo, M., \& Soares, P. (2018). Selenium and selenoproteins in immune mediated thyroid disorders. Diagnostics, 8(4), 70. https://doi.org/10.3390/diagnostics8040070

Silvah, J. H., de Lima, C. M. M., Nicoletti, C. F., Barbosa, A. C., Junqueira, G. P., da Cunha, S. F. D. C., \& Marchini, J. S. (2021). Protein provision and lower mortality in critically ill patients with COVID-19. Clinical Nutrition ESPEN, 45, 507-510. https://doi.org/10.1016/j.clnesp.2021.07.005

Tepasse, P. R., Vollenberg, R., Fobker, M., Kabar, I., Schmidt, H., Meier, J. A., \& Hüsing-Kabar, A. (2021). Vitamin A plasma levels in COVID-19 patients: a prospective multicenter study and hypothesis. Nutrients, 13(7), 2173. https://doi.org/10.3390/nu13072173

Thibault, R., Seguin, P., Tamion, F., Pichard, C., \& Singer, P. (2020). Nutrition of the COVID-19 patient in the intensive care unit (ICU): a practical guidance. Critical care, 24(1), 1-8. https://doi.org/10.1186/s13054-020-03159-z

Zhang, J., Rao, X., Li, Y., Zhu, Y., Liu, F., Guo, G., \& Peng, Z. (2021). Pilot trial of high-dose vitamin C in critically ill COVID-19 patients. Annals of intensive care, 11(1), 1-12. https://doi.org/10.1186/s13613-020-00792-3

Zhou, P., Yang, X. L., Wang, X. G., Hu, B., Zhang, L., Zhang, W., \& Shi, Z. L. (2020). A pneumonia outbreak associated with a new coronavirus of probable bat origin. Nature, 579(7798), 270-273. https://doi.org/10.1038/s41586-020-2012-7 